\title{
ON PRINCIPAL EIGENVALUES FOR PERIODIC PARABOLIC STEKLOV PROBLEMS
}

\author{
T. GODOY, E. LAMI DOZO, AND S. PACZKA
}

Received 1 March 2002

Let $\Omega$ be a $C^{2+\gamma}$ domain in $\mathbb{R}^{N}, N \geq 2,0<\gamma<1$. Let $T>0$ and let $L$ be a uniformly parabolic operator $L u=\partial u / \partial t-\sum_{i, j}\left(\partial / \partial x_{i}\right)\left(a_{i j}\left(\partial u / \partial x_{j}\right)\right)+\sum_{j} b_{j}\left(\partial u / \partial x_{i}\right)+a_{0} u$, $a_{0} \geq 0$, whose coefficients, depending on $(x, t) \in \Omega \times \mathbb{R}$, are $T$ periodic in $t$ and satisfy some regularity assumptions. Let $A$ be the $N \times N$ matrix whose $i, j$ entry is $a_{i j}$ and let $v$ be the unit exterior normal to $\partial \Omega$. Let $m$ be a $T$-periodic function (that may change sign) defined on $\partial \Omega$ whose restriction to $\partial \Omega \times \mathbb{R}$ belongs to $W_{q}^{2-1 / q, 1-1 / 2 q}(\partial \Omega \times(0, T))$ for some large enough $q$. In this paper, we give necessary and sufficient conditions on $m$ for the existence of principal eigenvalues for the periodic parabolic Steklov problem $L u=0$ on $\Omega \times \mathbb{R},\langle A \nabla u, v\rangle=\lambda m u$ on $\partial \Omega \times \mathbb{R}, u(x, t)=u(x, t+T), u>0$ on $\Omega \times \mathbb{R}$. Uniqueness and simplicity of the positive principal eigenvalue is proved and a related maximum principle is given.

\section{Introduction}

Let $\Omega$ be a $C^{2+\gamma}$ and bounded domain in $\mathbb{R}^{N}, N \geq 2,0<\gamma<1$, let $T>0$, let $\left\{a_{i j}\right\}_{1 \leq i, j \leq N},\left\{b_{j}\right\}_{1 \leq j \leq N}$ be two families of real functions defined on $\Omega \times \mathbb{R}$ satisfying $a_{i j} \in C^{\gamma, \gamma / 2}(\bar{\Omega} \times \mathbb{R}), b_{j} \in C^{1}(\bar{\Omega} \times \mathbb{R}), a_{i j}=a_{j, i}$, and $\partial a_{i j} / \partial x_{i} \in C(\bar{\Omega} \times \mathbb{R})$ for $1 \leq i, j \leq N$. Assume also that

$$
\sum_{i, j} a_{i j}(x, t) \xi_{i} \xi_{j} \geq \alpha_{0}|\xi|^{2}
$$

for some positive constant $\alpha_{0}$ and all $(x, t) \in \bar{\Omega} \times \mathbb{R}, \xi=\left(\xi_{1}, \ldots, \xi_{N}\right) \in \mathbb{R}^{N}$ and that each $a_{i j}(x, t), b_{j}(x, t)$ is $T$ periodic in $t$. Let $A$ be the $N \times N$ matrix whose $i, j$ entry is $a_{i j}$, let $b=\left(b_{1}, \ldots, b_{N}\right)$, let $a_{0}$ be a nonnegative and $T$ periodic function 
belonging to $C^{\gamma, \gamma / 2}(\bar{\Omega} \times \mathbb{R})$ and let $L$ be the parabolic operator given by

$$
L u=u_{t}-\operatorname{div}(A \nabla u)+\langle b, \nabla u\rangle+a_{0} u
$$

where $\langle$,$\rangle denotes the standard inner product on \mathbb{R}^{N}$.

For $q \geq 1, \tau>0$, let $W_{q}^{2,1}(\Omega \times(0, \tau))$ be the Sobolev space of the functions $u \in$ $L^{q}(\Omega \times(0, \tau)), \quad u=u(x, t), \quad x=\left(x_{1}, \ldots, x_{N}\right)$ such that $\partial u / \partial t, \partial u / \partial x_{j}$, and $\partial^{2} u / \partial x_{i} \partial x_{j}$ belong to $L^{q}(\Omega \times(0, \tau))$ for $1 \leq i, j \leq N$. We are interested in the periodic parabolic Steklov eigenvalue problem

$$
\begin{gathered}
L u=0 \quad \text { in } \Omega \times \mathbb{R}, \\
\langle A \nabla u, v\rangle=\lambda m u \quad \text { on } \partial \Omega \times \mathbb{R}, \\
u(x, t) \quad T \text { periodic in } t,
\end{gathered}
$$

where $v$ denotes the unit exterior normal to $\partial \Omega$ and the solution $u$ is taken such that $\left.u\right|_{\Omega \times(0, T)} \in W_{q}^{2,1}(\Omega \times(0, T))$ for a fixed and large enough $q$. The weight function $m$ is assumed $T$ periodic such that $\left.m\right|_{\partial \Omega \times(0, T)} \in W_{q}^{2-1 / q, 1-1 / 2 q}(\partial \Omega \times$ $(0, T)$ ) (the fractional Sobolev space defined, for example, as in [7, Chapter 2, paragraph 3]).

Steklov introduced this eigenvalue problem in the elliptic case in connection with the study of the map, nowadays called Dirichlet to Neumann map (cf. [3, Part B, Chapter VI, pages 395-404]) which is also of interest in the inverse problem of reconstructing the coefficients of $L$ from this map.

We say that $\lambda^{*} \in \mathbb{R}$ is a principal eigenvalue for the weight $m$ if (1.3) has a positive (i.e., a nonnegative and nontrivial) solution.

In this paper, we give necessary and sufficient conditions, on a weight $m$ as above, for existence of a positive principal eigenvalue. Uniqueness and simplicity of this positive principal eigenvalue is proved and a related form of the maximum principle is given.

We remark that this weighted eigenvalue problem includes the corresponding elliptic case where the coefficients are time independent.

In Section 2, for given $T$ periodic functions $f$ and $\Phi$ defined on $\Omega \times \mathbb{R}$ and $\partial \Omega \times \mathbb{R}$, respectively, and satisfying $\left.f\right|_{\partial \Omega \times(0, T)} \in L^{q}(\Omega \times(0, T)),\left.\Phi\right|_{\partial \Omega \times(0, T)} \in$ $W_{q}^{2-1 / q, 1-1 / 2 q}(\partial \Omega \times(0, T))$, we study existence of $T$ periodic solutions $u: \Omega \times$ $\mathbb{R} \rightarrow \mathbb{R}$, such that $\left.u\right|_{\partial \Omega \times(0, T)} \in W_{q}^{2,1}(\Omega \times \mathbb{R})$ for the problem

$$
\begin{gathered}
L u=f \quad \text { on } \Omega \times \mathbb{R}, \\
b_{0} u+\langle A \nabla u, v\rangle=\Phi \quad \text { on } \partial \Omega \times \mathbb{R}, \\
u(x, t) \quad T \text { periodic in } t .
\end{gathered}
$$

We prove that, under suitable hypothesis on $a_{0}$ and $b_{0}$, this problem has a unique solution, and we state the boundedness (with respect to the natural topologies 
involved) of the corresponding solution operator $u=S_{b_{0}}(f, \Phi)$ (see Theorem $2.5)$. We also prove the compactness and the strong positivity of the operator $\Phi \rightarrow S_{b_{0}}(0, \Phi)$ (see Theorem 2.6).

In Section 3, we study the following one-parameter family of principal eigenvalue problems: given $\lambda \in \mathbb{R}$, we prove that there exists a unique principal eigenvalue $\mu=\mu_{m}(\lambda)$ for the problem

$$
\begin{gathered}
L u=0 \quad \text { on } \Omega \times \mathbb{R}, \\
\langle A \nabla u, v\rangle-\lambda m u=\mu u \quad \text { on } \partial \Omega \times \mathbb{R}, \\
u(x, t) \quad T \text { periodic in } t, \\
u>0 \quad \text { on } \Omega \times \mathbb{R},
\end{gathered}
$$

we show that $\mu_{m}(\lambda)$ is concave and real analytic in $\lambda$ and its behavior at zero and at infinity is studied.

In Section 4, using the properties of the function $\mu_{m}$, we prove that, for the case $a_{0}>0$, the condition $P(m):=\int_{0}^{T} \max _{x \in \Omega} m(x, t) d t>0$ is a necessary and sufficient condition for the existence of a positive principal eigenvalue for the weighted problem (1.3) and that, for the case $a_{0}=0$, there exists a positive principal eigenvalue for (1.3) if and only if $P(m)>0$ and $\int_{\Omega \times(0, T)} \Psi m<0$ where $\Psi$ is a positive (unique up to a multiplicative constant and belonging to $C^{2+\gamma, 1+\gamma / 2}(\bar{\Omega} \times$ $\mathbb{R})$ ) for the $T$ periodic problem

$$
\begin{gathered}
\frac{\partial \Psi}{\partial t}+\operatorname{div}(A \nabla \Psi)+\langle b, \nabla \Psi\rangle+\operatorname{div}(b) \Psi=0 \quad \text { on } \Omega \times \mathbb{R} \\
\langle A \nabla \Psi, v\rangle+\langle b, v\rangle \Psi=0 \quad \text { on } \partial \Omega \times \mathbb{R} \\
\Psi(x, t) \quad T \text { periodic in } t
\end{gathered}
$$

\section{Preliminaries}

We recall the following well-known facts concerning Sobolev spaces (see, e.g., [7, Lemma 3.3, page 80 and Lemma 3.4, page 82]).

(i) If $u \in W_{q}^{2,1}(\Omega \times(0, \tau)), q \geq 1, \tau>0$, then

$$
\left.u\right|_{\partial \Omega \times(0, \tau)} \in W_{q}^{2-1 / q, 1-1 / 2 q}(\partial \Omega \times(0, \tau))
$$

and the restriction map (understood in the trace sense) is continuous.

(ii) For $\tau>0$ and $q$ large enough, it holds that

$$
W_{q}^{2,1}(\Omega \times(0, \tau)) \subset C^{1+\gamma,(1+\gamma) / 2}(\bar{\Omega} \times[0, \tau])
$$

with continuous inclusion. 
(iii) For $\tau>0$ and $q$ large enough, it holds that

$$
W_{q}^{2-1 / q, 1-1 / 2 q}(\partial \Omega \times(0, \tau)) \subset C^{1+\gamma,(1+\gamma) / 2}(\partial \Omega \times[0, \tau])
$$

with continuous inclusion.

From now on, we fix, $\tau>T$ and a large enough $q$ such that (ii) and (iii) hold.

We recall also the following lemma.

Lemma 2.1. Let $b_{0} \in W_{q}^{2-1 / q, 1-1 / 2 q}(\partial \Omega \times(0, \tau)), b_{0} \geq 0$. Suppose also that $f \in$ $L^{q}(\Omega \times(0, \tau)), \varphi \in W_{q}^{2-2 / q}(\Omega)$, and $\Phi \in W_{q}^{2-1 / q, 1-1 / 2 q}(\partial \Omega \times(0, \tau))$, and that the compatibility condition

$$
b_{0}(\cdot, 0) \varphi+\langle A \nabla \varphi, v\rangle=\Phi(\cdot, 0) \quad \text { on } \partial \Omega
$$

is fulfilled, then the problem

$$
\begin{aligned}
L u=f \quad \text { on } \Omega \times(0, \tau), & \\
b_{0} u+\langle A \nabla u, v\rangle=\Phi & \text { on } \partial \Omega \times(0, \tau), \\
u(\cdot, 0)=\varphi & \text { on } \Omega,
\end{aligned}
$$

has a unique solution $u \in W_{q}^{2,1}(\Omega \times(0, \tau))$. Moreover, there exists a positive constant $c$ independent of $f, \varphi$, and $\Phi$, such that

$$
\|u\|_{W_{q}^{2,1}(\Omega \times(0, T))} \leq c\left(\|f\|_{L^{q}(\Omega \times(0, T))}+\|\Phi\|_{W_{q}^{2-1 / q, 1-1 / 2 q}(\partial \Omega \times(0, T))}+\|\varphi\|_{W_{q}^{2-2 / q}(\Omega)}\right) .
$$

For a proof of Lemma 2.1, see [7, Theorem 9.1, page 341] concerning to the Dirichlet problem and its extension, to our boundary conditions, indicated there, at the end of Chapter 4, paragraph 9, page 351.

For regular data, the following result holds (see, e.g., [7, Theorem 5.3, page 320]).

Lemma 2.2. Suppose that $b_{0} \in C^{1+\gamma((1+\gamma) / 2)}(\partial \Omega \times[0, \tau]), b_{0} \geq 0$. Suppose also that $f \in C^{\gamma, \gamma / 2}(\bar{\Omega} \times[0, \tau]), \varphi \in C^{2+\gamma}(\bar{\Omega}), \Phi \in C^{1+\gamma((1+\gamma) / 2)}(\partial \Omega \times[0, \tau])$ and that the compatibility condition (2.4) is fulfilled, then problem (2.5) has a unique solution $u \in C^{2+\gamma, 1+\gamma / 2}(\bar{\Omega} \times[0, \tau])$. Moreover, there exists a positive constant $c$ independent of $f, \varphi$, and $\Phi$ such that

$$
\|u\|_{C^{2+\gamma, 1+\gamma / 2}(\bar{\Omega} \times[0, \tau])} \leq c\left(\|f\|_{C^{\gamma, \gamma / 2}(\bar{\Omega} \times[0, \tau])}+\|\Phi\|_{C^{1+\gamma((1+\gamma) / 2)}(\partial \Omega \times[0, \tau])}+\|\varphi\|_{C^{2+\gamma}(\bar{\Omega})}\right) .
$$

Remark 2.3. If, in addition to the hypothesis of Lemma 2.1, we have that $f \in$ $C^{\gamma, \gamma / 2}(\bar{\Omega} \times[0, \tau])$, then the solution $u$ of $(2.5)$ satisfies

$$
u \in C^{2+\gamma, 1+\gamma / 2}(\bar{\Omega} \times[\delta, \tau])
$$


for all $\delta>0$. Moreover, for such a $\delta$, there exists a positive constant $c_{\delta}$ independent of $f$ and $\Phi$ such that

$$
\|u\|_{C^{2+\gamma, 1+\gamma / 2}(\bar{\Omega} \times[\delta, \tau])} \leq c_{\delta}\left(\|f\|_{C^{\gamma, \gamma / 2}(\bar{\Omega} \times[0, \tau])}+\|\Phi\|_{W_{q}^{2-1 / q, 1-1 / 2 q}(\partial \Omega \times(0, \tau))}+\|\varphi\|_{W_{q}^{2-2 / q}(\Omega)}\right) .
$$

Indeed, let $h \in C^{\infty}(\mathbb{R})$ such that $0 \leq h \leq 1, h(t)=0$ for $t<\delta / 4, h(t)=1$ for $t \geq 3 \delta / 4$, let $\tilde{u}(x, t)=u(x, t) h(t)$, let $\tilde{f}(x, t)=u(x, t) h^{\prime}(t)+f(x, t) h(t)$, and let $\tilde{\Phi}(x, t)=\Phi(x, t) h(t)$. Then,

$$
\begin{array}{cc}
L \tilde{u}=\tilde{f} \quad \text { on } \Omega \times(0, \tau), & \\
b_{0} \tilde{u}+\langle A \nabla \tilde{u}, v\rangle=\tilde{\Phi} & \text { on } \partial \Omega \times(0, \tau), \\
\tilde{u}(\cdot, 0)=0 & \text { on } \Omega .
\end{array}
$$

By Lemma 2.1, this problem has a unique solution in $W_{q}^{2,1}(\Omega \times(0, \tau))$. Since $f \in C^{\gamma, \gamma / 2}(\bar{\Omega} \times[0, \tau])$ and $\tilde{\Phi} \in C^{1+\gamma,(1+\gamma) / 2}(\partial \Omega \times[0, \tau])$, Lemma 2.2 says that it has also a unique solution $\tilde{u} \in C^{2+\gamma,(1+\gamma) / 2}(\bar{\Omega} \times[0, \tau])$, and so, since $h \equiv 1$ on $[\delta, \tau]$, we obtain $(2.8)$.

Also,

$$
\|\tilde{\Phi}\|_{C^{1+\gamma,(1+\gamma) / 2}(\partial \Omega \times[0, T])} \leq c_{\delta}^{\prime}\|\Phi\|_{W_{q}^{2-1 / q, 1-1 / 2 q}(\partial \Omega \times(0, \tau))}
$$

for some constant $c_{\delta}^{\prime}$ independent of $\Phi$, and so, using (2.6) and the definition of $\tilde{f}$, we get

$$
\|\tilde{f}\|_{C^{\gamma, \gamma / 2}(\bar{\Omega} \times[0, \tau])} \leq c_{\delta}^{\prime \prime}\left(\|f\|_{C^{\gamma, \gamma / 2}(\bar{\Omega} \times[0, \tau])}+\|\Phi\|_{W_{q}^{2-1 / q, 1-1 / 2 q}(\partial \Omega \times(0, \tau))}+\|\varphi\|_{W_{q}^{2-2 / q}(\Omega)}\right)
$$

for some positive constant $c_{\delta}^{\prime \prime}$ independent of $f$, $\Phi$. Then (2.7), applied to problem (2.10), gives (2.9).

Let $b_{0} \in W_{q}^{2-1 / q, 1-1 / 2 q}(\partial \Omega \times(0, \tau)), b_{0} \geq 0$. For $s>1+1 / q$, let $W_{q, B_{0}}^{s}(\Omega)$ be the Banach space of the functions $\varphi \in W_{q}^{s}(\Omega)$ satisfying $b_{0}(\cdot, 0) \varphi+\langle A(\cdot, 0) \nabla \varphi, v\rangle=$ 0 on $\partial \Omega$.

$W_{q, B_{0}}^{2-2 / q}(\Omega)$ and $W_{q}^{2-1 / q, 1-1 / 2 q}(\partial \Omega \times(0, \tau))$ provided with their natural orders are ordered Banach spaces. Enlarging $q$, if necessary, we can assume (from now on) that in both spaces the respective positive cones have nonempty interior.

As usual, for $f: \Omega \times \mathbb{R} \rightarrow \mathbb{R}$ (resp., $f: \partial \Omega \times \mathbb{R} \rightarrow \mathbb{R}, f: \Omega \rightarrow \mathbb{R}$ ) we write $f>0$ to mean $f(x, t) \geq 0$ and $f$ nonidentically zero.

Let $U: W_{q, B_{0}}^{2-2 / q}(\Omega) \rightarrow W_{q, B_{0}}^{2-2 / q}(\Omega)$ be defined by $U \varphi=u(\cdot, T)$, where $u \in$ $W_{q}^{2,1}(\Omega \times(0, \tau))$ is the solution (given by Lemma 2.1) of

$$
\begin{aligned}
L u=0 & \text { on } \Omega \times(0, \tau), \\
b_{0} u+\langle A \nabla u, v\rangle=0 & \text { on } \partial \Omega \times(0, \tau), \\
u(\cdot, 0)=\varphi & \text { on } \Omega .
\end{aligned}
$$


We have the following lemma.

Lemma 2.4. Suppose that $b_{0} \in W_{q}^{2-1 / q, 1-1 / 2 q}(\partial \Omega \times(0, \tau)), b_{0} \geq 0$. Then $U$ is $a$ compact operator. Moreover, if either $a_{0}>0$ or $b_{0}>0$ in their respective domains, then $U$ is a strongly positive operator with positive spectral radius $\rho<1$.

Proof. From Lemma 2.1, the solution $u$ of (2.13) satisfies

$$
\|u\|_{W_{q}^{2,1}(\Omega \times(0, T))} \leq c\|\varphi\|_{W_{q}^{2-2 / q}(\Omega)} .
$$

Let $h, \tilde{u}, \tilde{f}$, and $\tilde{\Phi}$ be as in Remark 2.3, taking there $f=0, \Phi=0$. From (2.14) and (2.2), we have $\|\tilde{f}\|_{C^{\gamma, \gamma / 2}(\bar{\Omega} \times[0, T])} \leq c\|\varphi\|_{W_{q}^{2-2 / q}(\Omega)}$, and so, (2.9) applied to (2.13) implies

$$
\|u(\cdot, T)\|_{C^{2+\gamma}(\bar{\Omega})} \leq c\|\varphi\|_{W_{q}^{2-2 / q}(\Omega)}
$$

for some positive constant $c$ independent of $\varphi$. Now, (2.15) implies the compactness assertion of the lemma.

Suppose now that for some $\varphi>0$ in $W_{q, B_{0}}^{2-2 / q}(\Omega)$, the minimum of $U \varphi=$ $u(\cdot, T)$ is nonpositive. Then the minimum of $u$ on $\bar{\Omega} \times(0, T)$ is nonpositive and it is achieved at some $\left(x_{0}, t_{0}\right) \in \bar{\Omega} \times(0, T]$. If $x_{0} \in \Omega$, the parabolic maximum principle (as stated, e.g., in [6, Proposition 13.3, page 33]) implies that $u$ is a constant on $\bar{\Omega} \times[\delta, T]$ for all $\delta>0$, so $\varphi$ is a nonpositive constant which is a contradiction. If $x_{0} \in \partial \Omega$, the same principle states that $\langle A \nabla u, v\rangle<0$ at $\left(x_{0}, t_{0}\right)$ contradicting $b_{0}\left(x_{0}, t_{0}\right) u\left(x_{0}, t_{0}\right)+\langle A \nabla u, v\rangle\left(x_{0}, t_{0}\right)=0$. So, $U$ is a strongly positive operator on $W_{q}^{2-2 / q}(\Omega)$. Now, Krein-Rutman theorem (as stated, e.g., in [1, Theorem 3.1]) gives that its spectral radius $\rho$ is a positive eigenvalue with positive eigenfunctions. Let $\varphi_{\rho} \in W_{q}^{2-2 / q}(\Omega)$ be such an eigenfunction. To see that $\rho<1$, we proceed by contradiction. Suppose $\rho \geq 1$. Then $U\left(\varphi_{\rho}\right)=\rho \varphi_{\rho} \geq \varphi_{\rho}$, that is, the solution of (2.13) (assuming by taking $\varphi=\varphi_{\rho}$ ) would satisfy $u(\cdot, T) \geq \varphi_{\rho}$, but the maximum principle states that $u$ is a constant or $\max _{\bar{\Omega} \times[\delta, T]} u(x, t)$ is attained at some $\left(x_{0}, t_{0}\right) \in \partial \Omega \times[0, T]$ and so $a_{0}=0$ or $b_{0}\left(x_{0}, t_{0}\right)<0$, respectively.

Let $W_{q, T}^{2,1}(\Omega \times \mathbb{R})\left(\right.$ resp., $\left.W_{q, T}^{2-1 / q, 1-1 / 2 q}(\partial \Omega \times \mathbb{R})\right)$ the Banach space of the $T$ periodic functions $v: \Omega \times \mathbb{R} \rightarrow \mathbb{R}$ such that $\left.v\right|_{\Omega \times(0, T)} \in W_{q}^{2,1}(\Omega \times(0, T)$ ) (resp., $\left.v \mid \partial \Omega \times(0, T) \in W_{q}^{2-1 / q, 1-1 / 2 q}(\partial \Omega \times(0, T))\right)$, equipped with the norm $\|v\|_{W_{q}^{2,1}(\Omega \times(0, T))}$ (resp., $\left.\|v\|_{W_{q}^{2,1}(\partial \Omega \times(0, T))}\right)$.

Theorem 2.5. Let $b_{0}, \Phi \in W_{q, T}^{2-1 / q, 1-1 / 2 q}(\partial \Omega \times \mathbb{R}), b_{0} \geq 0$. If $a_{0}>0$ or $b_{0}>0$ and if $f: \Omega \times \mathbb{R} \rightarrow \mathbb{R}$ is $T$ periodic and satisfies $\left.f\right|_{\Omega \times(0, T)} \in L^{q}(\Omega \times(0, T))$, then the problem

$$
\begin{gathered}
L u=f \quad \text { on } \Omega \times \mathbb{R}, \\
b_{0} u+\langle A \nabla u, v\rangle=\Phi \quad \text { on } \partial \Omega \times \mathbb{R}, \\
u(x, t) \quad T \text { periodic in } t,
\end{gathered}
$$


has a unique solution $u \in W_{q, T}^{2,1}(\Omega \times \mathbb{R})$. Moreover, there exists a positive constant $c$ independent of $f$ and $\Phi$ such that

$$
\|u\|_{W_{q}^{2,1}(\Omega \times(0, T))} \leq c\left(\|\Phi\|_{W_{q}^{2-1 / q, 1-1 / 2 q}(\partial \Omega \times(0, T))}+\|f\|_{L^{q}(\Omega \times(0, T))}\right) .
$$

If in addition to the above hypothesis, $f \in C^{\gamma, \gamma / 2}(\bar{\Omega} \times \mathbb{R})$, then $u \in C^{2+\gamma, 1+\gamma / 2}(\bar{\Omega} \times$ $\mathbb{R})$ and

$$
\|u\|_{C^{2+\gamma, 1+\gamma / 2}(\bar{\Omega} \times \mathbb{R})} \leq c\left(\|\Phi\|_{W_{q}^{2-1 / q, 1-1 / 2 q}(\partial \Omega \times(0, T))}+\|f\|_{C^{\gamma, \gamma / 2}(\bar{\Omega} \times \mathbb{R})}\right)
$$

for some constant $c$ independent of $f$ and $\Phi$.

Proof. We start constructing a function $\varphi_{1} \in W^{2-2 / q}(\Omega)$ satisfying

$$
b_{0}(\cdot, 0) \varphi_{1}+\left\langle A(\cdot, 0) \nabla \varphi_{1}, v\right\rangle=\Phi(\cdot, 0)
$$

and such that

$$
\left\|\varphi_{1}\right\|_{W^{2-2 / q}(\Omega)} \leq \mathcal{c}\|\Phi\|_{W_{q}^{2-1 / q, 1-1 / 2 q}(\partial \Omega \times(0, T))}
$$

for some constant $c$ independent of $\Phi$. To do so, consider $F(\bar{x}, s)=\bar{x}-s A(\bar{x}, 0) v(\bar{x})$ on $\partial \Omega \times(-\varepsilon, \varepsilon)$. Since $\bar{x}(x)=x$ for $x \in \partial \Omega$ and $A(\bar{x}, 0) v(\bar{x})$ is nontangential to $\partial \Omega$ at $\bar{x} \in \partial \Omega, F$ defines a diffeomorphism onto a neighborhood $V$ of $\partial \Omega$ in $\mathbb{R}^{N}$ for some $\varepsilon>0$. So we have $F^{-1}(x)=(\bar{x}(x), s(x))$ for $x \in V$.

Then we solve the (noncharacteristic) Cauchy problem

$$
\begin{gathered}
b_{0}(\bar{x}(x), 0) w+\langle A(\bar{x}(x), 0) \nabla w, v(\bar{x}(x))\rangle=\Phi(\bar{x}(x), 0), \quad x \in V, \\
w=0 \quad \text { on } \partial \Omega,
\end{gathered}
$$

the solution is, for $x=\bar{x}-s A(\bar{x}, 0) v(\bar{x})$,

$$
w(x)=\Phi(\bar{x}, 0) \int_{0}^{s} e^{b_{0}(\bar{x}, 0)(\eta-s)} d \eta, \quad x \in V .
$$

Thanks to a cut-off function $h$ associated to $V$, we can extend $w$ to $\Omega$ by $\varphi_{1}=$ $h w$ which satisfies (2.19) and (2.20).

Let $u_{1} \in W^{2,1}(\Omega \times(0, \tau))$ be the solution, given by Lemma 2.1 , of the problem

$$
\begin{array}{cl}
L u_{1}=f \quad \text { on } \Omega \times(0, \tau), & \\
b_{0} u_{1}+\left\langle A \nabla u_{1}, v\right\rangle=\Phi & \text { on } \partial \Omega \times(0, \tau), \\
u_{1}(\cdot, 0)=\varphi_{1} & \text { on } \Omega .
\end{array}
$$

Thus, taking into account (2.20) and the estimate given by Lemma 2.1, we obtain

$$
\left\|u_{1}\right\|_{W_{q}^{2,1}(\Omega \times(0, T))} \leq c\left(\|\Phi\|_{W_{q}^{2-1 / q, 1-1 / 2 q}(\partial \Omega \times(0, T))}+\|f\|_{L^{q}(\Omega \times(0, T))}\right) .
$$


Since $b_{0}$ is $T$ periodic, $u_{1}(\cdot, T)-u_{1}(\cdot, 0) \in W_{q, B_{0}}^{2-2 / q}(\Omega)$. Let $\varphi_{2} \in W_{q, B_{0}}^{2-2 / q}(\Omega)$ be defined by

$$
\varphi_{2}=(I-U)^{-1}\left(u_{1}(\cdot, T)-u_{1}(\cdot, 0)\right)
$$

From (2.24), we get

$$
\left\|\varphi_{2}\right\|_{W_{q}^{2-2 / q}(\Omega)} \leq c\left(\|\Phi\|_{W_{q}^{2-1 / q, 1-1 / 2 q}(\partial \Omega \times(0, T))}+\|f\|_{L^{q}(\Omega \times(0, T))}\right)
$$

with the constant $c$ independent of $\Phi$ and $f$. Let $u_{2} \in W_{q}^{2,1}(\Omega \times(0, T))$ be the solution of the problem

$$
\begin{gathered}
L u_{2}=0 \quad \text { on } \Omega \times(0, \tau), \\
b_{0} u_{2}+\left\langle A \nabla u_{2}, v\right\rangle=0 \quad \text { on } \partial \Omega \times(0, \tau), \\
u(\cdot, 0)=\varphi_{2} \quad \text { on } \Omega,
\end{gathered}
$$

taking into account (2.26), Lemma 2.1 gives

$$
\left\|u_{2}\right\|_{W_{q}^{2,1}(\Omega \times(0, T))} \leq c\left(\|\Phi\|_{W_{q}^{2-1 / q, 1-1 / 2 q}(\partial \Omega \times(0, T))}+\|f\|_{L^{q}(\Omega \times(0, T))}\right) .
$$

Thus, $u:=u_{1}+u_{2}$ solves (2.16) on $\Omega \times(0, \tau)$. From (2.25), $u$ satisfies $u(\cdot, 0)=$ $u(\cdot, T)$. Also, (2.24) and (2.28) give (2.18). Moreover, it is easy to see that $u(x, t)-$ $u(x, t+T)$ is identically zero for $0 \leq t \leq \tau-T$. So, the $T$ periodic extension of $u$ (still denoted by $u$ ) solves (2.16) on $\Omega \times \mathbb{R}$. The uniqueness assertion of the lemma follows easily from the maximum principle.

Observe also that if $f \in C^{\gamma, \gamma / 2}(\Omega \times \mathbb{R})$, then, taking into account Remark 2.3, the periodicity of $u$ implies that $u \in C^{2+\gamma, 1+\gamma / 2}(\Omega \times \mathbb{R})$. From (2.17), we have

$$
\|u(\cdot, 0)\|_{W_{q}^{2-1 / q}(\Omega)} \leq c\left(\|\Phi\|_{W_{q}^{2-1 / q, 1-1 / 2 q}(\partial \Omega \times(0, T))}+\|f\|_{C^{\gamma, \gamma / 2}(\Omega \times \mathbb{R})}\right),
$$

and so, Remark 2.3, applied to (2.16), gives

$$
\|u(\cdot, T)\|_{C^{2+\gamma}(\bar{\Omega})} \leq c\left(\|\Phi\|_{W_{q}^{2-1 / q, 1-1 / 2 q}(\partial \Omega \times(0, T))}+\|f\|_{C^{\gamma, \gamma / 2}(\Omega \times \mathbb{R})}\right) .
$$

So, by the periodicity of $u$, the same estimate holds for $u(\cdot, 0)$. Then, (2.18) follows from the estimate given in Lemma 2.2.

Theorem 2.6. Let $a_{0}, b_{0}$, and $\Phi$ be as in Theorem 2.5 and let

$$
S_{b_{0}}: W_{q, T}^{2-1 / q, 1-1 / 2 q}(\partial \Omega \times \mathbb{R}) \longrightarrow W_{q, T}^{2-1 / q, 1-1 / 2 q}(\partial \Omega \times \mathbb{R})
$$


be the operator defined by $S_{b_{0}} \Phi=\left.u\right|_{\partial \Omega \times \mathbb{R}}$, where $u$ is the $T$ periodic solution of

$$
\begin{gathered}
L u=0 \quad \text { on } \Omega \times \mathbb{R}, \\
b_{0} u+\langle A \nabla u, v\rangle=\Phi \quad \text { on } \partial \Omega \times \mathbb{R}, \\
u(x, t) \quad T \text { periodic in } t,
\end{gathered}
$$

given by Theorem 2.5. Then $S_{b_{0}}$ is a compact strongly positive operator.

Proof. Theorem 2.5 gives

$$
\|u\|_{C^{2+\gamma, 1+\gamma / 2}(\bar{\Omega} \times[0, T])} \leq c\|\Phi\|_{W_{q}^{2-1 / q, 1-1 / 2 q}(\partial \Omega \times(0, T))} .
$$

From this estimate, the compactness of $S_{b_{0}}$ follows and, taking into account the regularity of the solution of (2.32), the assertion about the strong positivity of $S_{b_{0}}$ follows easily from the stated hypothesis on $a_{0}$ and $b_{0}$ and the maximum principle.

Corollary 2.7. Let $a_{0}, b_{0}, S_{b_{0}}$ be as in Theorem 2.6 and let $\rho$ be the spectral radius of $S_{b_{0}}$. Then, $\rho$ is positive and it is an algebraically simple eigenvalue of $S_{b_{0}}$ with positive associated eigenfunctions. Moreover, no other eigenvalue of $S_{b_{0}}$ has positive eigenfunctions associated.

Proof. The proof follows from Theorem 2.6 and the Krein-Rutman theorem.

\section{A one-parameter eigenvalue problem}

Let $m \in W_{q, T}^{2-1 / q, 1-1 / 2 q}(\partial \Omega \times \mathbb{R})$ be fixed from now on. In order to study principal eigenvalues for the weighted problem (1.3), we can assume, without loss of generality, that $\|m\|_{\infty} \leq 1 / 2$.

For $\varepsilon$ positive and small enough (i.e., such that $1-\varepsilon\left(1-\|m\|_{\infty}\right)>0$ ) and $\lambda>-\varepsilon$, let

$$
S_{\lambda, m}: W_{q, T}^{2-1 / q, 1-1 / 2 q}(\partial \Omega \times \mathbb{R}) \longrightarrow W_{q, T}^{2-1 / q, 1-1 / 2 q}(\partial \Omega \times \mathbb{R})
$$

be the operator defined by $S_{\lambda, m} \Phi=\left.u\right|_{\partial \Omega \times \mathbb{R}}$, where $u \in W_{q, T}^{2,1}(\Omega \times \mathbb{R})$ is the solution of the problem

$$
\begin{gathered}
L u=0 \quad \text { on } \Omega \times \mathbb{R}, \\
u+\lambda(1-m) u+\langle A \nabla u, v\rangle=\Phi \quad \text { on } \partial \Omega \times \mathbb{R}, \\
u(x, t) \quad T \text { periodic in } t,
\end{gathered}
$$

and let $\mu_{m}(\lambda)$ be defined by

$$
\frac{1}{1+\lambda+\mu_{m}(\lambda)}=\rho_{\lambda, m}
$$

where $\rho_{\lambda, m}$ is the spectral radius of $S_{\lambda, m}$. 
Remark 3.1. From Corollary 2.7, it follows that $\mu_{m}(\lambda)$ can be characterized as the unique real number $\mu$, such that problem

$$
\begin{gathered}
L u_{\lambda}=0 \quad \text { on } \Omega \times \mathbb{R}, \\
\left\langle A \nabla u_{\lambda}, v\right\rangle=\lambda m u_{\lambda}+\mu u_{\lambda} \quad \text { on } \partial \Omega \times \mathbb{R}, \\
u_{\lambda}(x, t) \quad T \text { periodic in } t
\end{gathered}
$$

has a positive solution $u_{\lambda} \in W_{q, T}^{2,1}(\Omega \times \mathbb{R})$. Since $\lambda m=(-\lambda)(-m)$, the above characterization of $\mu_{m}(\lambda)$ implies that $\mu_{m}(-\lambda)=\mu_{-m}(\lambda)$ for $\lambda \in(-\varepsilon, \varepsilon)$. We extend $\mu_{m}$ to the whole real line setting $\mu_{m}(-\lambda)=\mu_{-m}(\lambda)$ and so the above characterization of $\mu_{m}(\lambda)$ holds for all $\lambda \in \mathbb{R}$. In particular, this fact implies that $\mu_{m+k}(\lambda)=\mu_{m}(\lambda)-k \lambda$ for all $\lambda, k \in \mathbb{R}$.

Note also that, for fixed $\lambda \in \mathbb{R}$, the solution space in $W_{q, T}^{2,1}(\Omega \times \mathbb{R})$ of the problem

$$
\begin{gathered}
L u=0 \quad \text { on } \Omega \times \mathbb{R}, \\
\langle A \nabla u, v\rangle=\lambda m u+\mu_{m}(\lambda) u \quad \text { on } \partial \Omega \times \mathbb{R}, \\
u(x, t) \quad T \text { periodic in } t,
\end{gathered}
$$

is one dimensional and is contained in $C^{2+\gamma, 1+\gamma / 2}(\bar{\Omega} \times \mathbb{R})$. Moreover, by Corollary 2.7, positive solutions have a positive minimum on $\bar{\Omega} \times \mathbb{R}$.

From the above characterization of $\mu_{m}(\lambda)$, our problem (1.3) on principal eigenvalues is equivalent to find the zeroes of the function $\mu_{m}$.

Lemma 3.2. Suppose that $v \in C^{2+\gamma, 1+\gamma / 2}(\bar{\Omega} \times \mathbb{R})$, such that

$$
\begin{gathered}
L v \geq 0 \quad \text { on } \Omega \times \mathbb{R}, \\
\langle A \nabla v, v\rangle \geq \lambda m v+\bar{\mu} v \quad \text { on } \partial \Omega \times \mathbb{R}, \\
v>0 \quad \text { on } \Omega \times \mathbb{R}, \\
v(x, t) \quad \text { T periodic in } t,
\end{gathered}
$$

for some $\lambda, \bar{\mu} \in \mathbb{R}$. Then $\mu_{m}(\lambda) \geq \bar{\mu}$. If in addition either $L v>0$ or $\langle A \nabla v, v\rangle>$ $\lambda m v+\bar{\mu} v$, then $\mu_{m}(\lambda)>\bar{\mu}$.

Proof. We proceed by contradiction. Suppose that $\mu_{m}(\lambda)<\bar{\mu}$. From (3.6), we have, for $r$ large enough,

$$
\begin{gathered}
L v \geq 0 \quad \text { on } \Omega \times \mathbb{R}, \\
(r+\lambda(1-m)) v+\langle A \nabla v, v\rangle>\left(r+\lambda+\mu_{m}(\lambda)\right) v>0 \quad \text { on } \partial \Omega \times \mathbb{R}, \\
v(x, t) \quad T \text { periodic in } t .
\end{gathered}
$$

Then, the maximum principle implies that $v$ is bounded from below for some positive constant. Let $u_{\lambda}$ be a positive solution of (3.5). It follows that there exists a positive constant $c$, such that $u_{\lambda} \leq c v$ on $\bar{\Omega} \times \mathbb{R}$. Take $c$ minimal with respect to 
this property and let $w=c v-u_{\lambda}$. Then $\left.L w \geq 0,(r+\lambda(1-m)) w+\langle A \nabla w, v\rangle\right\rangle$ 0 on $\partial \Omega \times \mathbb{R}$. Now, the maximum principle implies that $\min _{\bar{\Omega} \times[0, T]} w>0$ and this leads to a contradiction with the choice of $c$. Finally, note that the above argument gives also the last assertion of the lemma.

Lemma 3.3. The function $\mu_{m}$ is a concave function.

Proof. Let $\lambda_{0}, \lambda_{1} \in \mathbb{R}$ and let $u_{\lambda_{0}}, u_{\lambda_{1}}$ be positive solutions of (3.5) for $\lambda=\lambda_{0}, \lambda_{1}$, respectively. For $\theta \in(0,1)$, let $u_{\theta}=u_{\lambda_{0}}^{\theta} u_{\lambda_{1}}^{1-\theta}$, so $u_{\theta} \in C^{2+\gamma, 1+\gamma / 2}(\bar{\Omega} \times \mathbb{R}), u_{\theta}$ is $T$ periodic and $u_{\theta}(x, t)>0$ for all $(x, t) \in \bar{\Omega} \times \mathbb{R}$. For $w \in \mathbb{R}^{N}$ and $(x, t) \in \Omega \times \mathbb{R}$, let $\|w\|_{A(x, t)}^{2}=\langle A(x, t) w, w\rangle$. We recall that for regular $u, v \in C^{2,1}(\Omega \times \mathbb{R}) \rightarrow \mathbb{R}$, such that $u(x, t)>0$ and $v(x, t)>0$ for all $(x, t) \in \Omega \times \mathbb{R}$ and for $\beta \in \mathbb{R}$ it holds that $L\left(u^{\beta}\right)=\beta u^{\beta-1} L u-\beta(\beta-1)|\nabla u|^{2} u^{\beta-2}$ and $L(u v)=u L v+v L u-2\langle A \nabla u, \nabla v\rangle$. Using these formulas and the definition of $\|\cdot\|_{A(x, t)}$, a direct computation shows that

$$
\left(L u_{\theta}\right)(x, t)=\theta(1-\theta)\left\|\left[\left(\frac{u_{\lambda_{1}}}{u_{\lambda_{0}}}\right)^{(1-\theta) / 2} \frac{\nabla u_{\lambda_{0}}}{u_{\lambda_{0}}^{1 / 2}}-\left(\frac{u_{\lambda_{0}}}{u_{\lambda_{1}}}\right)^{\theta / 2} \frac{\nabla u_{\lambda_{1}}}{u_{\lambda_{1}}^{1 / 2}}\right](x, t)\right\|_{A(x, t)}^{2}
$$

for $(x, t) \in \Omega \times \mathbb{R}$, and so, $L u_{\theta} \geq 0$ on $\Omega \times \mathbb{R}$. Another computation shows that

$$
\left\langle A \nabla u_{\theta}, v\right\rangle=\left(\theta \lambda_{0}+(1-\theta) \lambda_{1}\right) m u_{\theta}+\left(\theta \mu_{m}\left(\lambda_{0}\right)+(1-\theta) \mu_{m}\left(\lambda_{1}\right)\right) u_{\theta}
$$

on $\partial \Omega \times \mathbb{R}$. Then, this lemma follows from Lemma 3.2.

Remark 3.4. Lemma 3.3 implies that $\mu_{m}$ is continuous. Moreover, taking into account Corollary 2.7, we can apply [4, Lemma 1.3] (proceeding, e.g., as in [5, Remark 3.9 and Lemma 3.10]) to obtain that $\mu_{m}(\lambda)$ is real analytic in $\lambda$ for $\lambda>$ $-\varepsilon$ for some small enough positive $\varepsilon$, and since $\mu_{m}(-\lambda)=\mu_{-m}(\lambda)$ we get that $\mu_{m}$ is real analytic on the whole real line. Moreover, a positive solution $u_{\lambda}$ for (3.5) can be chosen such that $\lambda \rightarrow u_{\lambda \mid \partial \Omega \times(0, T)}$ is a real analytic map from $\mathbb{R}$ into $W_{q, T}^{2-1 / q, 1-1 / 2 q}(\partial \Omega \times \mathbb{R})$.

Note also that if $a_{0}=0$, then $\mu_{m}(0)=0$ and that, in this case, the eigenfunctions associated for (3.5) are the constant functions. Finally, for the case $a_{0}>0$, applying Lemma 3.2 with $\nu=1, \lambda=0$ and $\bar{\mu}=0$, we obtain that $\mu_{m}(0)>0$.

Lemma 3.5. Let $m_{1}, m_{2} \in W_{q, T}^{2-1 / q, 1-1 / 2 q}(\partial \Omega \times \mathbb{R})$. Suppose that $m_{1}<m_{2}$. Then, $\mu_{m_{1}}(\lambda)>\mu_{m_{2}}(\lambda)$ for all $\lambda>0$.

Proof. Since for $c \in \mathbb{R}-\{0\} \mu_{c m_{j}}(\lambda)=\mu_{m_{j}}(\lambda / c), j=1,2$, we can assume, without loss of generality, that $\left\|m_{j}\right\|_{\infty}<1 / 2, j=1,2$. For $\lambda>0$, let $S_{\lambda, m_{j}}$ be defined as before at the beginning of this section. Let $\Phi \in W_{q, T}^{2-1 / q, 1-1 / 2 q}(\partial \Omega \times \mathbb{R})$ such that $\Phi>0$, let $u_{j}=S_{\lambda, m_{j}} \Phi, j=1,2$ and let $v=u_{1}-u_{2}$. A computation shows that $v$ satisfies $L v=0$ on $\Omega \times \mathbb{R}$ and $\langle A \nabla v, v\rangle+\lambda\left(1-m_{1}\right) v=\lambda\left(m_{1}-m_{2}\right) v$ on $\partial \Omega \times \mathbb{R}$; thus, Theorem 2.6 implies $v<0$. Then, $S_{\lambda, m_{1}}<S_{\lambda, m_{2}}$, this gives $\rho_{\lambda, m_{1}}<\rho_{\lambda, m_{2}}$, and so, $\mu_{m_{1}}(\lambda)>\mu_{m_{2}}(\lambda)$. 
412 Principal eigenvalues for a Steklov problem

In order to make explicit the dependence on $L$, we denote by $S_{L, \lambda, m}$ the operator $S_{\lambda, m}$ as defined at the beginning of this section. We also denote by $\mu_{m, L}$ the function $\mu_{m}$. Let $L_{0}$ be the operator defined by $L_{0} u=\partial u / \partial t-\operatorname{div}(A \nabla u)+$ $\langle b, \nabla u\rangle$. We have the following lemma.

Lemma 3.6. Suppose that $a_{0} \neq 0$. Then $\mu_{m, L}(\lambda)>\mu_{m, L_{0}}(\lambda)$ for all $\lambda \in \mathbb{R}$.

Proof. Suppose that $\lambda \geq 0$. Let $\Phi \in W_{q, T}^{2-1 / q, 1-1 / 2 q}(\partial \Omega \times \mathbb{R})$, with $\Phi>0$, let $k>$ $\|m\|_{\infty}$, let $u=S_{L, \lambda, m} \Phi$, let $u_{0}=S_{L_{0}, \lambda, m} \Phi$ and let $v=u-u_{0}$. Then $L_{0} v=-a_{0} u<0$ on $\Omega \times \mathbb{R},(\lambda(k-m)+1) v+\langle A \nabla v, v\rangle=0$ on $\partial \Omega \times(0, T)$, and $v(x, t) T$ periodic in $t$. Thus the maximum principle gives $v \leq 0$. So $S_{L, \lambda, m}<S_{L_{0}, \lambda, m}$. This implies that $\mu_{m, L}(\lambda)>\mu_{m, L_{0}}(\lambda)$. Since $\mu_{m, L}(\lambda)=\mu_{-m, L}(-\lambda)$ (and similarly for $L_{0}$ ), the case $\lambda<0$ reduces to the previous one.

Remark 3.7. Suppose that $a_{0}=0$. Let $k \in \mathbb{R}, k>\sum_{1 \leq j \leq N}\left\|b_{j}\right\|_{\infty}$, let

$$
S_{k}: W_{q, T}^{2-1 / q, 1-1 / 2 q}(\partial \Omega \times \mathbb{R}) \longrightarrow W_{q, T}^{2-1 / q, 1-1 / 2 q}(\partial \Omega \times \mathbb{R})
$$

be defined by (2.17) and (2.18) taking $b_{0}=k$ and let $\rho_{k}$ be its spectral radius. Since $\Phi=1$ is a positive eigenfunction associated to the eigenvalue $1 / k$, the Krein-Rutman theorem asserts that $\rho_{k}=1 / k$. Thus, also by Krein-Rutman theorem, there exists a positive eigenvector $\Psi$ for the adjoint operator $S_{k}^{*}$ satisfying $S_{k}^{*} \Psi=\Psi$. Moreover, such a $\Psi$ is unique up to a multiplicative constant.

Lemma 3.8. Suppose that $a_{0}=0$ and let $S_{k}, \Psi$ be as in Remark 3.7. Then $\mu_{m}^{\prime}(0)=$ $-\langle\Psi, m\rangle /\langle\Psi, 1\rangle$.

Proof. For $\lambda \in \mathbb{R}$, let $u_{\lambda}$ be a solution of (3.5) such that $\lambda \rightarrow u_{\lambda}$ is real analytic and such that $u_{0}=1$

$$
\begin{gathered}
L u_{\lambda}=0 \quad \text { on } \Omega \times \mathbb{R}, \\
k u_{\lambda}+\left\langle A \nabla u_{\lambda}, v\right\rangle=\left(\lambda m+\mu_{m}(\lambda)+k\right) u_{\lambda} \quad \text { on } \partial \Omega \times \mathbb{R}, \\
u_{\lambda}(x, t) \quad T \text { periodic in } t,
\end{gathered}
$$

we get $u_{\lambda}=\lambda S_{k}\left(m u_{\lambda}\right)+\left(\mu_{m}(\lambda)+k\right) S_{k} u_{\lambda}$ and so

$$
\lambda\left\langle\Psi, m u_{\lambda}\right\rangle+\mu_{m}(\lambda)\left\langle\Psi, u_{\lambda}\right\rangle=0 .
$$

Taking the derivative with respect to $\lambda$ at $\lambda=0$ and using that $\mu_{m}(0)=0$ and that $u_{0}=1$, the lemma follows.

For $\Phi, f \in W_{q, T}^{2-1 / q, 1-1 / 2 q}(\partial \Omega \times \mathbb{R})$, let $\langle i(\Phi), f\rangle=\int_{\partial \Omega \times(0, T)} \Phi f$. So $i(\Phi) \in$ $\left(W_{q, T}^{2-1 / q, 1-1 / 2 q}(\partial \Omega \times \mathbb{R})\right)^{\prime}$. We have the following lemma. 
Lemma 3.9. Suppose that $a_{0}=0$ and let $k, S_{k}, \Psi$ be as in Remark 3.7. Then,

(i) for $f \in W_{q, T}^{2-1 / q, 1-1 / 2 q}(\partial \Omega \times \mathbb{R})$, we have $S_{k}^{*} f=i\left(\left.v\right|_{\partial \Omega \times \mathbb{R}}\right)$, where $v$ is the $T$ periodic solution of the problem

$$
\begin{gathered}
\frac{\partial v}{\partial t}+\operatorname{div}(A \nabla v)+\langle b, \nabla v\rangle+\operatorname{div}(b) v=0 \quad \text { on } \Omega \times \mathbb{R} \\
\langle A \nabla v, v\rangle+(k+\langle b, v\rangle) v=f \quad \text { on } \partial \Omega \times \mathbb{R}, \\
v(x, t) \quad T \text { periodic in } t
\end{gathered}
$$

(ii) $\Psi \in C^{2+\gamma, 1+\gamma / 2}(\bar{\Omega} \times \mathbb{R})$ and $\min _{\bar{\Omega} \times \mathbb{R}} \Psi>0$. Moreover, $\Psi$ can be characterized as the (unique up to a multiplicative constant) solution of the $T$ periodic problem

$$
\begin{gathered}
\frac{\partial \Psi}{\partial t}+\operatorname{div}(A \nabla \Psi)+\langle b, \nabla \Psi\rangle+\operatorname{div}(b) \Psi=0 \quad \text { on } \Omega \times \mathbb{R}, \\
\langle A \nabla \Psi, v\rangle+\langle b, v\rangle \Psi=0 \quad \text { on } \partial \Omega \times \mathbb{R}, \\
\Psi(x, t) \quad T \text { periodic in } t .
\end{gathered}
$$

Proof. Note that, for $f \in W_{q, T}^{2-1 / q, 1-1 / 2 q}(\partial \Omega \times \mathbb{R}),(3.13)$ has a unique $T$ periodic solution $v \in C^{2+\gamma, 1+\gamma / 2}(\bar{\Omega} \times \mathbb{R})$. Indeed, the change of variable $t=T-\tau$ reduces (3.13) to the situation studied in Theorem 2.5. In order to prove part (i) of the lemma, we must show that $\langle i(v), \Phi\rangle=\int_{\partial \Omega \times(0, T)} S(\Phi) f$, that is,

$$
\int_{\partial \Omega \times(0, T)} v \Phi=\int_{\partial \Omega \times(0, T)} f u,
$$

where $u$ is the $T$ periodic solution of the problem

$$
\begin{gathered}
\frac{\partial u}{\partial t}-\operatorname{div}(A \nabla u)+\langle b, \nabla u\rangle=0 \quad \text { on } \Omega \times \mathbb{R}, \\
k u+\langle A \nabla u, v\rangle=\Phi \quad \text { on } \partial \Omega \times \mathbb{R}, \\
u(x, t) \quad T \text { periodic in } t .
\end{gathered}
$$

Multiplying (3.13) by $u$, (3.16) by $v$, adding, and integrating on $\Omega \times(0, T)$, we get

$$
\begin{aligned}
0=\int_{\Omega \times(0, T)} \frac{\partial(u v)}{\partial t}+\int_{\Omega \times(0, T)}[ & \operatorname{div}(u A \nabla v)-\operatorname{div}(v A \nabla u) \\
& +v\langle b, \nabla u\rangle+u\langle b, \nabla v\rangle+u v \operatorname{div}(b)] .
\end{aligned}
$$

The first integral vanishes by the periodicity. Taking into account the boundary conditions of (3.13) and (3.16), an application of the divergence theorem gives (3.15). To prove (ii), consider the operator

$$
\tilde{S}: W_{q, T}^{2-1 / q, 1-1 / 2 q}(\partial \Omega \times \mathbb{R}) \longrightarrow W_{q, T}^{2-1 / q, 1-1 / 2 q}(\partial \Omega \times \mathbb{R}),
$$


defined by $\tilde{S} f=\left.v\right|_{\partial \Omega \times \mathbb{R}}$, where $v$ is the solution of (3.13). Note that, via the change of variable $t=T-\tau$, Theorem 2.6 gives that $\widetilde{S}$ is a compact and strongly positive operator. Thus, $\tilde{S}$ has a positive spectral radius which is an eigenvalue with an associated positive $T$ periodic eigenfunction $h$, that, by Theorem 2.5 , belongs to $C^{2+\gamma, 1+\gamma / 2}(\bar{\Omega} \times \mathbb{R})$. Moreover, $\min _{\bar{\Omega} \times \mathbb{R}} h>0$. Let $\Psi$ be as in Remark 3.7. By Lemma 3.9, $h$ is a positive eigenvector for $S^{*}$ and so, by Krein-Rutman theorem, we get $\Psi=c h$ for some positive constant $c>0$. Thus (ii) holds.

We set

$$
\begin{aligned}
P(m) & =\int_{0}^{T} \max _{x \in \partial \Omega} m(x, t) d t, \\
N(m) & =\int_{0}^{T} \min _{x \in \partial \Omega} m(x, t) d t .
\end{aligned}
$$

Proceeding as in [2], it can be shown that if $P(m)>0$, then there exists a $T$ periodic curve $\Gamma \in C^{2}(\mathbb{R}, \partial \Omega)$, such that

$$
\int_{0}^{T} m(\Gamma(t), t) d t>0
$$

we fix, from now on, such a $\Gamma$.

For $p \in \partial \Omega$, let $T_{p}(\partial \Omega)$ denotes the tangent space to $\partial \Omega$ at $p$ and let $\exp _{p}$ : $T_{p}(\partial \Omega) \rightarrow T_{p}(\partial \Omega)$ be the geodesic exponential map defined by $\exp _{p}(X)=\sigma_{p, X}(1)$, where $\sigma_{p, X}$ is the geodesic in $\partial \Omega$ (respect to the natural Riemannian structure on $\partial \Omega$ inherit from $\left.\mathbb{R}^{N}\right)$ satisfying $\sigma_{p, X}(0)=p,(d / d s)\left(\sigma_{p, X}(s)\right)=X$. Since $\partial \Omega$ is of class $C^{2+\gamma}, \exp _{p}$ is a well-defined map.

Lemma 3.10. For $\delta$ positive and small enough, there exists

$$
\Lambda \in C^{1}\left((-\delta, \delta)^{N} \times \mathbb{R}, \mathbb{R}^{N+1}\right)
$$

such that $\Lambda$ is a diffeomorphism from $(-\delta, \delta)^{N} \times \mathbb{R}$ onto an open neighborhood $W_{\delta} \subset \mathbb{R}^{N} \times \mathbb{R}$ of the set $\{(T(t), t): t \in \mathbb{R}\}$ satisfying

(1) $\Lambda\left((-\delta, \delta)^{N-1} \times(0, \delta) \times \mathbb{R}\right)=W_{\delta} \cap(\Omega \times \mathbb{R})$,

(2) $\Lambda\left((-\delta, \delta)^{N-1} \times\{0\} \times \mathbb{R}\right)=W_{\delta} \cap(\partial \Omega \times \mathbb{R})$,

(3) $\Lambda(0, t)=(\Gamma(t), t)$,

(4) $\Lambda(\cdot, t)$ is $T$ periodic in $t$.

Moreover, $\Lambda:(-\delta, \delta)^{N} \times \mathbb{R} \rightarrow W_{\delta}$ and its inverse $\Theta: W_{\delta} \rightarrow(-\delta, \delta)^{N} \times \mathbb{R}$ are of class $C^{2,1}$ on their respective domains.

Proof. The map $t \rightarrow v(\Gamma(t))$ is $T$ periodic and belongs to the class $C^{1+\gamma}\left(\mathbb{R}, \mathbb{R}^{N}\right)$. Then, there exists a $C^{1+\gamma}$ and $T$ periodic map $t \rightarrow A(t)$ from $\mathbb{R}$ into $\mathrm{SO}(N)$ such that $A(t) v(\Gamma(0))=v(\Gamma(t)), t \in \mathbb{R}$. Let $\left\{X_{1,0}, \ldots, X_{N-1,0}\right\}$ be a basis of $T_{\Gamma(0)}(\partial \Omega)$ and let $X_{j}(t)=A(t) X_{j, 0}, j=1,2, \ldots, N-1$. Thus, each $X_{j}$ is a $T$ periodic map, 
$X_{j} \in C^{1+\gamma}\left(\mathbb{R}, \mathbb{R}^{N}\right)$ and for each $t,\left\{X_{1}(t), \ldots, X_{N-1}(t)\right\}$ is a basis of $T_{\Gamma(t)}(\partial \Omega)$. For $\delta$ positive and small enough, and for $(s, t) \in(-\delta, \delta)^{N} \times \mathbb{R}$, let

$$
x(s, t)=\exp _{\Gamma(t)}\left(\sum_{1 \leq j \leq N-1} s_{j} X_{j}(t)\right)-s_{N} v\left(\exp _{\Gamma(t)}\left(\sum_{1 \leq j \leq N-1} s_{j} X_{j}(t)\right)\right),
$$

and let

$$
\Lambda(s, t)=(x(s, t), t) .
$$

From the well-known properties of the exponential map, it follows easily that, for $\delta$ small enough, $(s, t) \rightarrow \Lambda(s, t)$ is a $C^{2,1}$ map which satisfies the properties required by the lemma.

Let $\delta, \Lambda, \Theta, W_{\delta}$ be as in Lemma 3.10, $\Theta(x, t)=\left(\Theta_{1}(x, t), \ldots, \Theta_{N+1}(x, t)\right)$. Note that, since $\Theta_{N}$ vanishes identically on $W_{\delta} \cap(\partial \Omega \times \mathbb{R})$, we have

$$
\nabla \Theta_{N}=-g v \quad \text { on } W_{\delta} \cap(\partial \Omega \times \mathbb{R})
$$

for some $g \in C^{1}\left(W_{\delta} \cap(\partial \Omega \times \mathbb{R})\right)$ satisfying $g(x, t) \neq 0$ for all $(x, t) \in W_{\delta} \cap(\partial \Omega \times$ $\mathbb{R})$. Moreover,

$$
\Theta^{\prime}(\Gamma(t), t) \Lambda^{\prime}(0, t)=\mathrm{Id}
$$

(where $\Lambda^{\prime}$ and $\Theta^{\prime}$ denote the respective $(N+1) \times(N+1)$ Jacobian matrix of $\Lambda$ and $\Theta$, respectively). Thus, considering the $(N, N)$ entries in this equality and using (3.25) and that $\left(\partial \Lambda_{N}\right) /\left(\partial s_{N \mid(0, t)}\right)=-v(\Gamma(t))$, we get

$$
g(\Gamma(t), t)=1, \quad \forall t \in \mathbb{R} .
$$

Lemma 3.11. Suppose that $P(m)>0$, then $\lim _{\lambda \rightarrow \infty} \mu_{m}(\lambda)=-\infty$.

Proof. Let $\delta, \Lambda, \Theta, W_{\delta}$ be as in Lemma 3.10. Let $Q_{T, \delta}=(-\delta, \delta)^{N-1} \times[0, \delta) \times$ $(0, T)$ and let $D_{T, \delta}=\Lambda\left(Q_{T, \delta}\right) \subset \bar{\Omega} \times(0, T)$. If $f: D_{\delta} \rightarrow \mathbb{R}$ (resp., $f: D_{\delta} \cap(\partial \Omega \times$ $\mathbb{R}) \rightarrow \mathbb{R}$ ) let $f^{\#}: Q_{T, \delta} \rightarrow \mathbb{R}\left(\right.$ resp., $\left.f^{\#}:(-\delta, \delta)^{N-1} \times\{0\} \times(0, T) \rightarrow \mathbb{R}\right)$ be defined by $f^{\#}=f \circ \Lambda$.

For $\lambda>0$, let $u_{\lambda} \in C^{2+\gamma, 1+\gamma / 2}(\bar{\Omega} \times \mathbb{R})$ be a positive $T$ periodic solution of (3.5), since $u_{\lambda}=u_{\lambda}^{\#} \circ \Theta$ on $D_{\delta}$, the equation $L u_{\lambda}=0$ on $D_{\delta}$ gives

$$
\frac{\partial u_{\lambda}^{\#}}{\partial t}-\operatorname{div}\left(A^{\#} \nabla u_{\lambda}^{\#}\right)+\left\langle b^{\#}, \nabla u_{\lambda}^{\#}\right\rangle+a_{0}^{\#} u_{\lambda}^{\#}=0 \quad \text { on } Q_{T, \delta},
$$

where $A^{\#}$ is the $N \times N$ symmetric and positive matrix whose $(i, j)$ entry is

$$
a_{i j}^{\#}(s, t)=\sum_{1 \leq l, r \leq N} a_{l r}(\Lambda(s, t)) \frac{\partial \Theta_{i}}{\partial x_{l}}(\Lambda(s, t)) \frac{\partial \Theta_{j}}{\partial x_{r}}(\Lambda(s, t))
$$


and where $b^{\#}=\left(b_{1}^{\#}, \ldots, b_{N}^{\#}\right)$ with each $b_{j}^{\#}$ belonging to $C\left(Q_{T, \delta}, \mathbb{R}\right)$ and independent of $\lambda$.

If $v(x, t)=\left(v_{1}(x, t), \ldots, v_{N}(x, t)\right)$, the boundary condition

$$
\left\langle A \nabla u_{\lambda}, v\right\rangle=\lambda m u_{\lambda}+\mu_{m}(\lambda) u_{\lambda} \quad \text { on }(\partial \Omega \times(0, T)) \cap D_{\delta}
$$

transforms into

$$
\begin{array}{r}
\sum_{1 \leq i, j, l \leq N} a_{i j}(\Lambda(s, t)) \frac{\partial u_{\lambda}^{\#}}{\partial s_{l}}(s, t) \frac{\partial \Theta_{l}}{\partial x_{j}}(\Lambda(s, t)) v_{i}(\Lambda(s, t)) \\
=\lambda m^{\#}(s, t) u_{\lambda}^{\#}(s, t)+\mu_{m}(\lambda) u_{\lambda}^{\#}(s, t)
\end{array}
$$

for all $(s, t) \in(-\delta, \delta)^{N-1} \times\{0\} \times(0, T)$.

Let $g$ be given by (3.25). Taking into account (3.29) and (3.25) from (3.31), we get

$$
\left\langle A^{\#} \nabla u_{\lambda}^{\#}, e_{N}\right\rangle=-\lambda m^{\#} g^{\#} u_{\lambda}^{\#}-\mu_{m}(\lambda) g^{\#} u_{\lambda}^{\#} \quad \text { on }(-\delta, \delta)^{N-1} \times\{0\} \times(0, T),
$$

where $\nabla$ denotes the gradient in the variables $s_{1}, \ldots, s_{N}$ and $e_{N}=(0, \ldots, 0,1)$.

Note that $\int_{0}^{T} m^{\#}(0, t) d t=\int_{0}^{T} m(\Lambda(0, t)) d t=\int_{0}^{T} m(\Gamma(t), t) d t>0$ and thus, by (3.28), $g^{\#}(0, t)=1$. Since $m^{\#}$ and $g^{\#}$ are continuous on $(-\delta, \delta)^{N-1} \times\{0\} \times \mathbb{R}$, we have, for $\eta$ small enough

$$
\begin{aligned}
& \int_{0}^{T} \int_{\left\{\sigma \in \mathbb{R}^{N-1}:|\sigma|<\eta\right\}}\left(m^{\#} g^{\#}\right)(\sigma, 0, t) d \sigma d t>0, \\
& \int_{0}^{T} \int_{\left\{\sigma \in \mathbb{R}^{N-1}:|\sigma|<\eta\right\}} g^{\#}(\sigma, 0, t) d \sigma d t>0 .
\end{aligned}
$$

Let $\beta \in(0, \eta)$, let $h \in C^{\infty}(\mathbb{R})$, such that $0 \leq h \leq 1, h(\zeta)=1$ for $\zeta<\eta-\beta, h(\zeta)=0$ for $\zeta \geq \eta$ and let $G: \mathbb{R}^{N+1} \rightarrow \mathbb{R}$ be defined by $G(s, t)=h(|s|)$; thus, $G \in C^{\infty}\left(\mathbb{R}^{N+1}\right)$. From (3.33) and (3.34), it is easy to see that we can pick $\beta$ small enough such that

$$
\begin{aligned}
& \int_{0}^{T} \int_{\left\{\sigma \in \mathbb{R}^{N-1}:|\sigma|<\eta\right\}}\left(G^{2} m^{\#} g^{\#}\right)(\sigma, 0, t) d \sigma d t>0, \\
& \int_{0}^{T} \int_{\left\{\sigma \in \mathbb{R}^{N-1}:|\sigma|<\eta\right\}}\left(G^{2} g^{\#}\right)(\sigma, 0, t) d \sigma d t>0 .
\end{aligned}
$$

Let $B_{\eta, T}=\left\{(s, t) \in \mathbb{R}^{N} \times(0, T):|s|<\eta, s_{N} \geq 0\right\}$. We multiply (3.28) by $G^{2} / u_{\lambda}^{\#}$ and then, integrating on $B_{\eta, T}$ and taking into account that $u_{\lambda}^{\#}(\cdot, 0)=u_{\lambda}^{\#}(\cdot, T)$ and that $G$ does not depend on $t$, we get

$$
\int_{B_{\eta, T}}\left[-\frac{G^{2}}{u_{\lambda}^{\#}} \operatorname{div}\left(A^{\#} \nabla u_{\lambda}^{\#}\right)+\frac{G^{2}}{u_{\lambda}^{\#}}\left\langle b^{\#}, \nabla u_{\lambda}^{\#}\right\rangle+a_{0}^{\#} G^{2}\right]=0 .
$$


Let $v_{\lambda}^{\#}=-\log u_{\lambda}^{\#}$. Thus $v_{\lambda}^{\#} \in C^{2,1}\left(B_{\eta, T}\right)$. A computation gives that

$$
\begin{aligned}
-\frac{G^{2}}{u_{\lambda}^{\#}} \operatorname{div}\left(A^{\#} \nabla u_{\lambda}^{\#}\right)= & \operatorname{div}\left(G^{2} A^{\#} \nabla v_{\lambda}^{\#}\right)-2\left\langle A^{\#} G \nabla v_{\lambda}^{\#}, \nabla G\right\rangle \\
& -\left\langle A^{\#} G \nabla v_{\lambda}^{\#}, G \nabla v_{\lambda}^{\#}\right\rangle \quad \text { on } B_{\eta, T} .
\end{aligned}
$$

Also,

$$
\frac{G^{2}}{u_{\lambda}^{\#}}\left\langle b^{\#}, \nabla u_{\lambda}^{\#}\right\rangle=-2\left\langle G A^{\#} \nabla v_{\lambda}^{\#}, \frac{1}{2} G\left(A^{\#}\right)^{-1} b^{\#}\right\rangle \quad \text { on } B_{\eta, T},
$$

so, from (3.40) the divergence theorem gives

$$
\begin{aligned}
\int_{0}^{T} \int_{\left\{\sigma \in \mathbb{R}^{N-1}:|\sigma|<\eta\right\}} G^{2}\left\langle A^{\#} \nabla v_{\lambda}^{\#}, v\right\rangle= & -2 \int_{B_{\eta, T}}\left\langle G A^{\#} \nabla v_{\lambda}^{\#}, \nabla G+\frac{1}{2} G\left(A^{\#}\right)^{-1} b^{\#}\right\rangle \\
& +\int_{B_{\eta, T}}\left\langle A^{\#} G \nabla v_{\lambda}^{\#}, G \nabla v_{\lambda}^{\#}\right\rangle+\int_{B_{\eta, T}} a_{0}^{\#} G^{2} .
\end{aligned}
$$

For $w \in \mathbb{R}^{N}$ and $(s, t) \in B_{\eta, T}$, let $\|w\|_{A^{\#}(s, t)}=\left\langle A^{\#}(s, t) w, w\right\rangle$. Taking into account the boundary condition (3.33) and that $G(s)=0$ for $|s|=\eta$ from (3.40), we get

$$
\begin{aligned}
\mu_{m}(\lambda) \int_{0}^{T} \int_{\left\{\sigma \in \mathbb{R}^{N-1}:|\sigma|<\eta\right\}}\left(G^{2} g^{\#}\right)(\sigma, 0, t) d \sigma d t \\
=-\lambda \int_{0}^{T} \int_{\left\{\sigma \in \mathbb{R}^{N-1}:|\sigma|<\eta\right\}}\left(G^{2} g^{\#} m^{\#}\right)(\sigma, 0, t) d \sigma d t \\
-\int_{B_{\eta, T}}\left\|\left(G \nabla v_{\lambda}^{\#}+\nabla G+\frac{1}{2} G\left(A^{\#}\right)^{-1} b^{\#}\right)(s, t)\right\|_{A^{\#}(s, t)}^{2} d s d t \\
+\int_{B_{\eta, T}}\left\|\left(\nabla G+\frac{1}{2} A^{\#} G b^{\#}\right)(s, t)\right\|_{A^{\#}(s, t)}^{2} d s d t+\int_{B_{\eta, T}} a_{0}^{\#} G^{2} \\
\leq-\lambda \int_{0}^{T} \int_{\left\{\sigma \in \mathbb{R}^{N-1}:|\sigma|<\eta\right\}}\left(G^{2} g^{\#} m^{\#}\right)(\sigma, 0, t) d \sigma d t \\
+\int_{B_{\eta, T}}\left\|\left(\nabla G+\frac{1}{2} A^{\#} G b^{\#}\right)(s, t)\right\|_{A^{\#}(s, t)}^{2} d s d t+\int_{B_{\eta, T}} a_{0}^{\#} G^{2} .
\end{aligned}
$$

From this inequality, (3.35), and (3.36), the lemma follows.

\section{Principal eigenvalues for periodic parabolic Steklov problems}

Let $P(m)$ and $N(m)$ be defined by (3.19) and (3.20), respectively. We have the following theorem.

Theorem 4.1. Suppose either $a_{0}>0$ and $P(m)>0$ (resp., $a_{0}>0$ and $N(m)<0$ ) or $a_{0}=0, P(m)>0$, and $\langle\Psi, m\rangle<0$ (resp., $a_{0}=0, N(m)<0$, and $\langle\Psi, m\rangle>0$ ) with 
$\Psi$ defined as in Remark 3.7. Then, there exists a unique positive (resp., negative) principal eigenvalue for (1.3) and the associated eigenspace is one dimensional.

Proof. Suppose $a_{0}=0$ and $P(m)>0,\langle\Psi, m\rangle<0$. Since $\mu_{m}(0)=0$ and, by Lemma $3.8, \mu_{m}^{\prime}(0)>0$ the existence of a positive principal eigenvalue $\lambda=\lambda_{1}(m)$ for $(1.3)$ follows from Lemma 3.11. Since $\mu_{m}$ does not vanish identically, the concavity of $\mu_{m}$ gives the uniqueness of the positive principal eigenvalue.

Moreover, if $u, v$ are solutions in $C^{2+\gamma, 1+\gamma / 2}(\bar{\Omega} \times \mathbb{R})$ for $(1.3)$, then, by the facts stated in Remark 3.1, $u=c v$ on $\partial \Omega \times \mathbb{R}$ for some constant $c$. Since $L(u-c v)=0$ on $\Omega \times \mathbb{R}, u-c v=0$ on $\partial \Omega \times \mathbb{R}$, and $u-c v$ is $T$ periodic, it follows easily from the maximum principle that $u=c v$ on $\Omega \times \mathbb{R}$.

If $a_{0}>0$, then (by Remark 3.4) $\mu_{m}(0)>0$; thus, the existence follows from Lemma 3.11. The other assertions of the theorem follows as in the case $a_{0}=$ 0 . Taking into account that $\mu_{m}(-\lambda)=\mu_{-m}(\lambda)$ and that $N(m)=-P(-m)$, the assertions about negative principal eigenvalues follow from the previous cases.

Lemma 4.2. Suppose that $a_{0}=0$. Then for all $\lambda>0$,

$$
\mu_{m}(\lambda) \geq-\frac{P(m)}{T} \lambda-\|b\|_{\infty}-\frac{|\Omega|}{|\partial \Omega|}\|\operatorname{div}(b)\|_{\infty} .
$$

Proof. We consider first the case $m \geq 0$. Let $\lambda>0$ and let $u_{\lambda}$ be a positive solution of (3.5) normalized by $\left\|u_{\lambda}\right\|_{\infty}=1$. From

$$
\begin{gathered}
\frac{\partial u_{\lambda}}{\partial t}-\operatorname{div}\left(A \nabla u_{\lambda}\right)+\left\langle b, \nabla u_{\lambda}\right\rangle=0 \quad \text { on } \Omega \times(0, T), \\
\left\langle A \nabla u_{\lambda}, v\right\rangle=\lambda m u_{\lambda}+\mu_{m}(\lambda) u_{\lambda} \quad \text { on } \partial \Omega \times(0, T), \\
u_{\lambda}(\cdot, 0)=u_{\lambda}(\cdot, T),
\end{gathered}
$$

and since $\left\langle b, \nabla u_{\lambda}\right\rangle=\operatorname{div}\left(u_{\lambda} b\right)-u_{\lambda} \operatorname{div}(b)$, integrating (4.2) on $\Omega \times(0, T)$ and taking into account the periodicity of $u_{\lambda}$ and the boundary conditions, the divergence theorem gives

$$
\mu_{m}(\lambda) \int_{\partial \Omega \times(0, T)} u_{\lambda}=-\lambda \int_{\partial \Omega \times(0, T)} m u_{\lambda}+\int_{\partial \Omega \times(0, T)} u_{\lambda}\langle b, v\rangle-\int_{\Omega \times(0, T)} u_{\lambda} \operatorname{div}(b) .
$$

Since $m \geq 0$ and $\left|u_{\lambda}\right| \leq 1$, we have $\int_{\partial \Omega \times(0, T)} m u_{\lambda} \leq P(m)|\partial \Omega|$, also $\left|u_{\lambda} \operatorname{div}(b)\right| \leq$ $\|\operatorname{div}(b)\|_{\infty}$ and $\left|u_{\lambda}\langle b, v\rangle\right| \leq\|b\|_{\infty}$. Thus

$$
\begin{aligned}
T|\partial \Omega| \mu_{m}(\lambda) & \geq \mu_{m}(\lambda) \int_{\partial \Omega \times(0, T)} u_{\lambda} \\
& \geq-\lambda P(m)|\partial \Omega|-T|\partial \Omega|\|b\|_{\infty}-\|\operatorname{div}(b)\|_{\infty}|\Omega| T,
\end{aligned}
$$

so the lemma holds for $m \geq 0$. For the general case, pick $k \in \mathbb{R}, k>\|m\|_{\infty}$ taking into account that $P(m+k)=P(m)+k T$ and that $\mu_{m+k}(\lambda)=\mu_{m}(\lambda)-k \lambda$ the lemma follows from the previous case applied to $m+k$ instead of $m$. 
Corollary 4.3. Suppose that $a_{0}=0$. Then, $\lim _{\lambda \rightarrow \infty} \mu_{m}^{\prime}(\lambda) \geq-P(m) / T$.

Proof. Suppose that $P(m) \neq 0$, then Lemmas 3.11 and 4.2 imply that $\lim _{\lambda \rightarrow \infty} \mu_{m}(\lambda)$ $= \pm \infty$. Also, $\mu_{m}$ is concave; thus, there exists $\lim _{\lambda \rightarrow \infty} \mu_{m}^{\prime}(\lambda)$. Then, the L'Hopital rule gives $\lim _{\lambda \rightarrow \infty} \mu_{m}^{\prime}(\lambda)=\lim _{\lambda \rightarrow \infty} \mu_{m}(\lambda) / \lambda \geq-P(m) / T$, the last inequality by Lemma 4.2. If $P(m)=0$ and if $\mu_{m}(\lambda)<0$ for some $\lambda>0$ then, since $\mu_{m}(0)=0$, the concavity of $\mu_{m}$ implies that $\lim _{\lambda \rightarrow \infty} \mu_{m}(\lambda)=-\infty$ and the above argument applies. If $\mu_{m}(\lambda) \geq 0$ for all $\lambda>0$, the concavity implies that $\mu_{m}^{\prime}(\lambda) \geq 0$ for all $\lambda>0$ and so the corollary is also true in this case.

Lemma 4.4. Suppose that $a_{0}=0$ and let $\Psi$ be as in Remark 3.7. Then, $P(m)<0$ implies that $\langle\Psi, m\rangle<0$.

Proof. Suppose $P(m)<0$. By Corollary 4.3, we have $\lim _{\lambda \rightarrow \infty} \mu_{m}^{\prime}(\lambda)>0$. Then, since $\mu_{m}$ is concave, we have $\mu_{m}^{\prime}(0)>0$ and so $\langle\Psi, m\rangle<0$.

Lemma 4.5. Suppose that $a_{0}=0$. Then, $\mu_{m}$ vanishes identically if and only if $P(m)=\langle\Psi, m\rangle=0$.

Proof. Suppose that $\mu_{n}$ vanishes identically. Lemma 3.8 gives that $\langle\Psi, m\rangle=0$. Also, by Lemma 3.11, we have $P(m) \leq 0$. Suppose that $P(m)<0$ and let $\tilde{m}(t)=$ $\max _{x \in \partial \Omega} m(x, t)$. Since $m \in C(\partial \Omega \times \mathbb{R})$, it follows easily that $\tilde{m} \in C[0, T]$. Take $\varepsilon$ such that $0<\varepsilon T<-P(m)$ and take a $T$ periodic function $m^{*} \in C^{1}(\mathbb{R})$ such that $\tilde{m}(t)<m^{*}(t)<\tilde{m}(t)+\varepsilon, t \in[0, T]$. Thus, $-\varepsilon T>P(m)=P(\tilde{m})>P\left(m^{*}\right)-$ $\varepsilon T$. Thus, $P\left(m^{*}\right)<0$ and so, by Lemma $2.4,\left\langle\Psi, m^{*}\right\rangle<0$. Thus $\mu_{m^{*}}^{\prime}(0)>0$ and then, since $m<m^{*}$, for $\lambda$ positive and small enough, we have $\mu_{m}(\lambda) \geq \mu_{m^{*}}(\lambda)>0$ contradicting our original assumption.

Suppose now that $P(m)=\langle\Psi, m\rangle=0$. Then $\mu_{m}^{\prime}(0)=0$ and also, by Corollary $4.3, \lim _{\lambda \rightarrow \infty} \mu_{m}^{\prime}(\lambda) \geq 0$. Then, the concavity of $\mu_{m}$ implies that $\mu_{m}^{\prime}$ vanishes identically on the positive axis, and so, since $\mu_{m}(0)=0$ the same is true for $\mu_{m}$ and since $\mu_{m}$ is analytic, vanishes on the whole line.

Theorem 4.6. Suppose that $a_{0}=0$ and that $\mu_{m}$ does not vanish identically. Then, the conditions $P(m)>0$ and $\langle\Psi, m\rangle<0$ (resp., $N(m)<0$ and $\langle\Psi, m\rangle>0$ ) are necessary for the existence of a positive (resp., negative) principal eigenvalue for (1.3).

Proof. Suppose that $\mu_{m}\left(\lambda_{1}\right)=0$ for some $\lambda_{1}>0$. Since $\mu_{m}(0)=0$ and $\mu_{m}$ is concave, we must have $\mu_{m}^{\prime}(0)>0$, and so, $\langle\Psi, m\rangle<0$. To see that $P(m)>0$, we proceed by contradiction. Suppose that $P(m) \leq 0$. Corollary 4.3 implies that $\lim _{\lambda \rightarrow \infty} \mu_{m}^{\prime}(\lambda) \geq 0$ and so, since $\mu_{m}$ is concave, we have $\mu_{m}^{\prime}(\lambda) \geq 0$ for all $\lambda>0$, and then, since $\mu_{m}^{\prime}(0)>0, \mu_{m}$ cannot vanish on the positive axis.

Theorem 4.7. Suppose that $a_{0}>0$. Then, the condition $P(m)>0$ (resp., $N(m)<$ 0 ) is necessary for the existence of a positive (resp., negative) principal eigenvalue for (1.3).

Proof. For $\lambda>0$, by Lemmas 3.5 and 3.6, we have $\mu_{m, L}(\lambda) \geq \mu_{\tilde{m}, L}(\lambda) \geq \mu_{\tilde{m}, L_{0}}(\lambda)$. Suppose that $P(m) \leq 0$. Corollary 4.3 gives $\lim _{\lambda \rightarrow \infty} \mu_{\tilde{m}, L_{0}}^{\prime}(\lambda) \geq 0$, and so, 
$\mu_{\tilde{m}, L_{0}}(\lambda) \geq 0$ for all $\lambda>0$. Since $\mu_{m}(0)>0$, the concavity of $\mu_{m}$ implies that $\mu_{m}$ cannot vanish on the positive axis.

Theorem 4.8. Let $\lambda \in \mathbb{R}$ such that $\mu_{m}(\lambda)>0$. Then, for all $h \in W_{q, T}^{2-1 / q, 1-1 / 2 q}(\partial \Omega \times$ $\mathbb{R})$, the problem

$$
\begin{gathered}
L u=0 \quad \text { in } \Omega \times \mathbb{R}, \\
\langle A \nabla u, v\rangle=\lambda m u+h \quad \text { on } \partial \Omega \times \mathbb{R}, \\
u(x, t) \quad T \text { periodic in } t,
\end{gathered}
$$

has a unique solution. Moreover, $h>0$ implies that $\min _{\bar{\Omega} \times(0, T)} u>0$.

Proof. Let $k, S_{\lambda, k, m}$, and $\rho_{\lambda, k, m}$ be as in Remark 3.1. Since $\mu_{m}(\lambda)>0$, we have $\rho_{\lambda, k, m}<1 /(\lambda k+1)$, and so, since $S_{\lambda, k, m}$ is a strongly positive operator, $((1 /(\lambda k+1)) I$ $\left.-S_{\lambda, k, m}\right)^{-1}$ is a well-defined and positive operator. Equation (4.5) is equivalent to $u=(\lambda k+1) S_{\lambda, k, m} u+S_{\lambda, k, m} h$, that is, to

$$
u=\frac{1}{\lambda k+1} S_{\lambda, k, m}\left(\frac{1}{\lambda k+1} I-S_{\lambda, k, m}\right)^{-1} h .
$$

So the theorem follows.

Let $\lambda_{1}(m)$ (resp., $\lambda_{-1}(m)$ ) be the positive (resp., negative) principal eigenvalue for the weight $m$ with the convention that $\lambda_{1}(m)=+\infty$ (resp., $\lambda_{-1}(m)=-\infty$ ) if there does not exist such a principal eigenvalue. From the properties of $\mu_{m}$ we obtain the following corollary as an immediate consequence of Theorem 4.8.

Corollary 4.9. Assume that $a_{0}>0$. Then, the interval $\left(\lambda_{-1}(m), \lambda_{1}(m)\right)$ does not contain eigenvalues for problem (1.3). If $a_{0}=0$, the same is true for the intervals $\left(\lambda_{-1}(m), 0\right)$ and $\left(0, \lambda_{1}(m)\right)$.

\section{Acknowledgment}

This work was partially supported by Agencia Cordoba Ciencia, Secyt-UNC, Secyt-UBA, and Conicet.

\section{References}

[1] H. Amann, Fixed point equations and nonlinear eigenvalue problems in ordered Banach spaces, SIAM Rev. 18 (1976), no. 4, 620-709.

[2] A. Beltramo and P. Hess, On the principal eigenvalue of a periodic-parabolic operator, Comm. Partial Differential Equations 9 (1984), no. 9, 919-941.

[3] S. Bergman and M. Schiffer, Kernel Functions and Elliptic Differential Equations in Mathematical Physics, Academic Press, New York, 1953.

[4] M. G. Crandall and P. H. Rabinowitz, Bifurcation, perturbation of simple eigenvalues and linearized stability, Arch. Rational Mech. Anal. 52 (1973), no. 2, 161-180.

[5] T. Godoy, E. Lami Dozo, and S. Paczka, The periodic parabolic eigenvalue problem with $L^{\infty}$ weight, Math. Scand. 81 (1997), no. 1, 20-34. 
[6] P. Hess, Periodic-Parabolic Boundary Value Problems and Positivity, Pitman Research Notes in Mathematics Series, vol. 247, Longman Scientific \& Technical, Harlow, 1991.

[7] O. A. Ladyženskaja, V. A. Solonnikov, and N. N. Ural'ceva, Linear and Quasilinear Equations of Parabolic Type, Translations of Mathematical Monographs, vol. 23, American Mathematical Society, Rhode Island, 1967.

T. Godoy: Facultad de Matemática, Astronomía y Física and CiEM - Conicet Universidad Nacional de Córdoba, Ciudad Universitaria, 5000 Córdoba, Argentina

E-mail address: godoy@mate.uncor.edu

E. Lami Dozo: Departement de Mathematique, Université Libre de Bruxelles, Campus Plaine 214, 1050 Bruxelles, Belgique

Current address: IAM-Conicet and Universidad de Buenos Aires SaAvedra 15, 3er Piso 1083 Buenos Aires, Argentina

E-mail address: lamidozo@ulb.ac.be

S. Paczka: Facultad de Matemática, Astronomía y Física and CieM - Conicet Universidad Nacional de Córdoba, Ciudad Universitaria, 5000 Córdoba, Argentina

E-mail address: paczka@mate. uncor.edu 


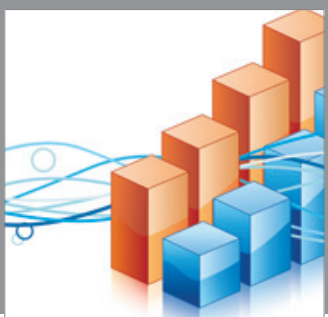

Advances in

Operations Research

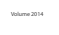

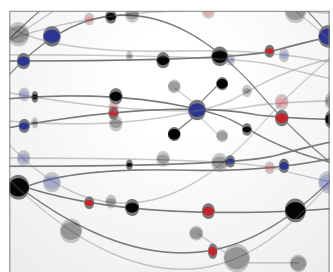

\section{The Scientific} World Journal
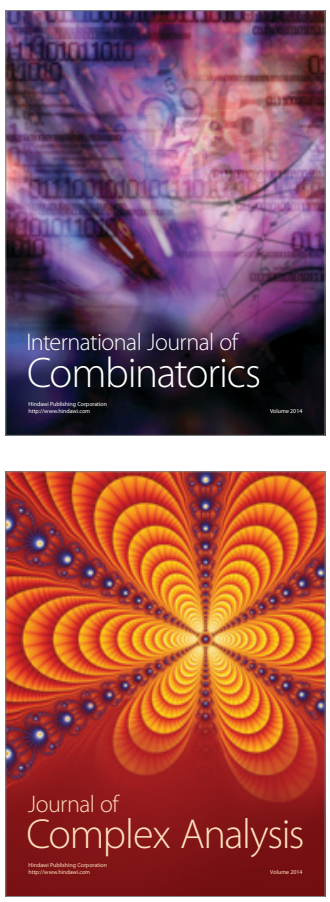

International Journal of

Mathematics and

Mathematical

Sciences
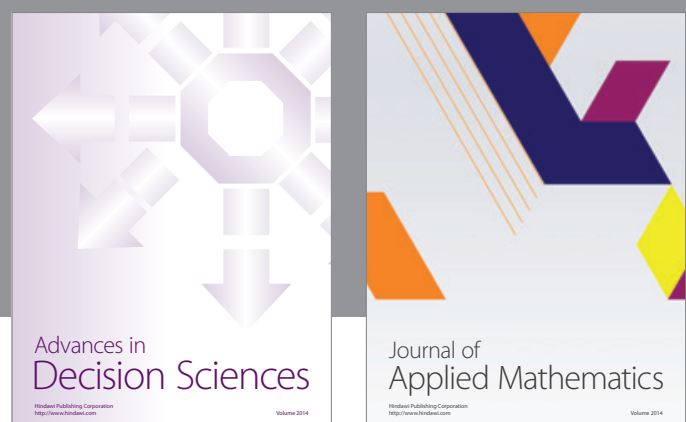

Journal of

Applied Mathematics
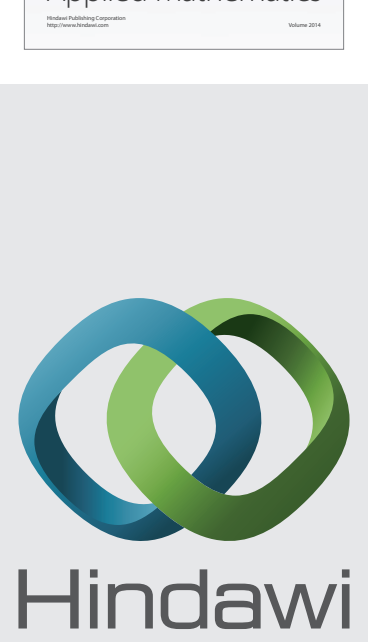

Submit your manuscripts at http://www.hindawi.com
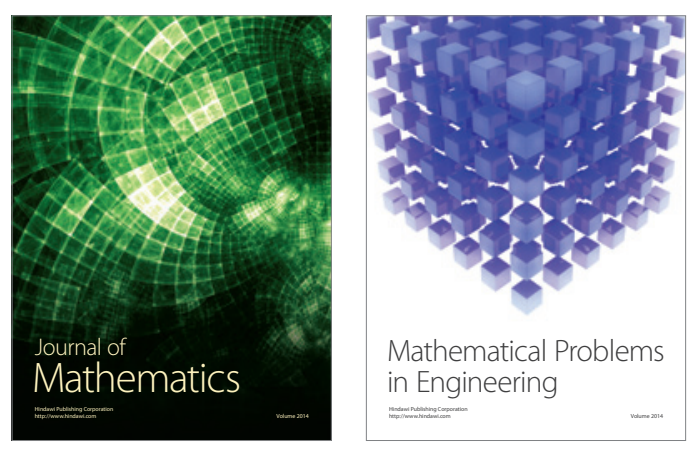

Mathematical Problems in Engineering
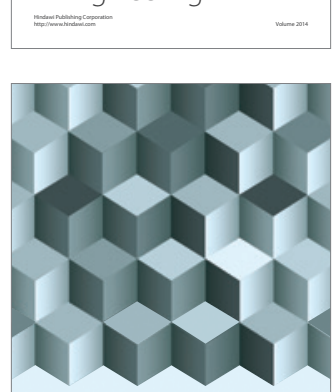

Journal of

Function Spaces
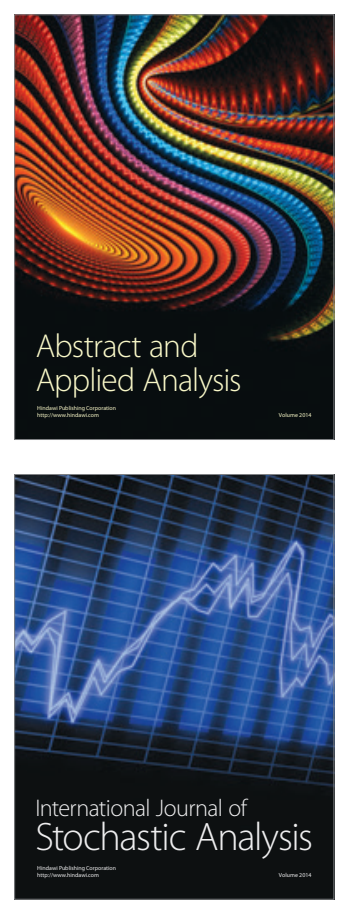

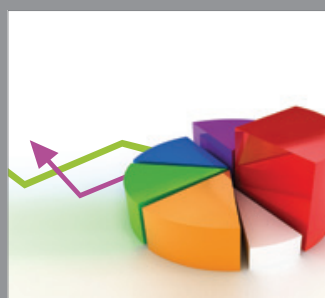

ournal of

Probability and Statistics

Promensencen
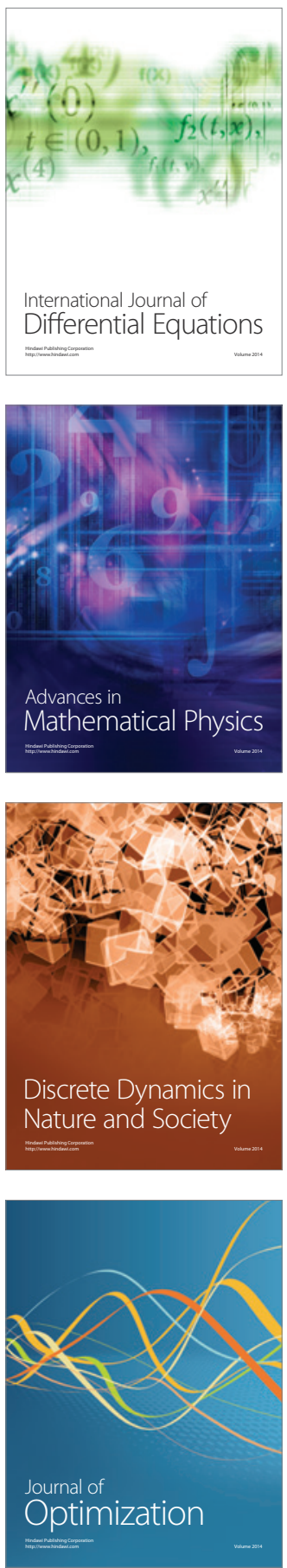